\title{
3 Research Square

\section{Randomized clinical trial to assess the protective efficacy of a Plasmodium vivax CS synthetic vaccine}

Socrates Herrera ( $\nabla$ sherrera@inmuno.org)

Caucaseco Scientific Research Center https://orcid.org/0000-0001-6354-0184

Myriam Arevalo-Herrera

Centro de Investigación Científica Caucaseco

Michelle Larmart-Delgado

Malaria Vaccine and Drug Development Center (MVDC)

Maria Alejandra Caicedo

Malaria Vaccine and Drug Development Center (MVDC)

Juliana Henao-Geraldo

Malaria Vaccine and Drug Development Center (MVDC)

Angelica Castellanos

Malaria Vaccine and Drug Development Center (MVDC)

Xiomara Gaitan

Malaria Vaccine and Drug Development Center (MVDC)

Sonia Herrera

Caucaseco Scientific Research Center

Jean-Christophe Devaud

Biochemistry Department, University of Lausanne

André Panatier

Biochemistry Department, University of Lausanne

Jose Oñate

Centro Médico Imbanaco

Giampietro Corradin

University of Lausanne

\section{Article}

Keywords: Plasmodium vivax, circumsporozoite protein, malaria, vaccines, clinical trial, Phase II trial, Colombia

Posted Date: April 9th, 2021 
DOI: https://doi.org/10.21203/rs.3.rs-374553/v1

License: (c) (1) This work is licensed under a Creative Commons Attribution 4.0 International License. Read Full License 


\section{Abstract}

A randomized, double-blind, controlled clinical trial was conducted to assess the safety and protective efficacy of the Plasmodium vivax circumsporozoite (CS) protein. A total of 35 healthy adults, either malaria-naïve $(n=17)$ or P.vivax semi-immune $(n=18)$, were enrolled and immunized intramuscularly (i.m.) at months 0 , 2, and 6, with the PvCS $(150 \mu \mathrm{g})$ formulated in Montanide ISA-51 adjuvant. Most volunteers developed PvCS specific antibody and T-cell responses and were subjected to a P. vivax sporozoite controlled human malaria infection (CHMI) 30 days after the last immunization. Sterile protection was observed in five of 11 naïve (42\%) and four of 11 semi-immune (36\%) volunteers by showing no parasitemia during the 60 days follow-up, as did a semi-immune control (1/5) volunteer. All non-protected volunteers developed malaria symptoms 10-19 days after $\mathrm{CHMI}$ and were immediately treated with antimalarial drugs. This is the first study of a P. vivax sub-unit vaccine demonstrating sterile protection against P. vivax infection.

\section{Introduction}

An estimated $\sim 229$ million malaria clinical cases and $>409,000$ deaths occurred worldwide in 2019, with a substantial economic impact on populations living in developing regions [1]. Epidemiological indicators have shown that $P$. vivax infections remain widely distributed worldwide, representing a significant cause of morbidity and mortality among 2.5 billion people living at risk of disease by this malaria parasite species [2]. Based on substantial evidence that supports their feasibility, vaccines are considered a critical tool to complement classical malaria control strategies that have not worked on their own [3]. $P$. falciparum RTS.S/AS01E, based on the CS protein use, is currently the most advanced malaria vaccine candidate with a mean efficacy of $\sim 34 \%$ in phase III trials [3-5]. It was approved by the European Medicines Agency (EMA) [6] and is currently undergoing implementation phase in several African countries [7]. A systematic analysis of the P. vivax CS (PVCS) antigen has been performed during the last 20 years [8]. After immunological characterization of the PvCS protein and identification of multiple Band T-cell epitopes [9], three long synthetic peptides (LSP) (N, R, and C) were designed, synthesized, and used in preclinical studies to test their immunogenicity in BALB/c mice and Aotus monkeys. These studies demonstrated high immunogenicity in both animal species $[9,10]$. Phase I clinical study was then conducted in 69 healthy malaria-naïve volunteers to evaluate the three PVCS LSP in a dose-escalating manner (10, 30, and 100 $\mathrm{g} /$ dose), formulated in Montanide ISA 720 [11]. This trial demonstrated good safety and tolerability, and high immunogenicity of the three individual fragments ( $N, R$, and $\mathrm{C}$-term). A second Phase I trial was further conducted in 40 volunteers who were vaccinated with mixtures of the three peptides formulated either in Montanide ISA-720 or Montanide ISA-51 at 50 and 100 $\mathrm{g} / \mathrm{dose}$ [12]. Vaccine formulations were well-tolerated, and no serious adverse events (SAE) were observed with any of the vaccines. All individuals seroconverted (ELISA) and developed antibodies to all three protein regions, which also recognized the native protein on $P$. vivax sporozoites as determined by indirect immunofluorescent antibody test (IFAT). Moreover, vaccine- antibodies inhibited $P$. vivax sporozoite 
invasion (ISI) to liver-cell lines [13]. Further studies allowed the standardization of a $P$. vivax sporozoite controlled human malaria infection (CHMI) in healthy and semi-immune volunteers [14-16].

Moreover, $P$. vivax CS formulations using recombinant products have been developed and tested by other groups in phase I trials, which demonstrated a good safety profile, although displayed low immunogenicity. First, the repeat region of the VK210 P. vivax variant was expressed in E. coli [17], formulated in Alum, and tested in 13 volunteers (doses of 10-1000 $\mu \mathrm{g} /$ dose); this protein displayed a good safety profile but low and no boostable antibody response detectable by ELISA. Second, a recombinant protein expressing $70 \%$ of the PVCS protein sequence was produced in yeast [18], formulated in Alum and tested in doses ranging from 50 to $400 \mu \mathrm{g} /$ dose $(n=30)$. Volunteers exposed to the higher doses (200-400 $\mu \mathrm{g} / \mathrm{dose}$ ) generated minimal humoral and cellular responses. More recently, a hybrid recombinant construct encompassing both VK210 and VK247 repeats alleles [19] was expressed in E. coli (VMP001), formulated in AS01B adjuvant, and evaluated in phase $\mathrm{I} /$ Ila vaccine dose escalation. Volunteers $(n=30)$ developed robust humoral and T-cell responses, and a slight delay in patency (1-2 days) was observed in $59 \%$ of the volunteers [20].

Herein, a phase lla/b clinical trial to evaluate the safety and protective efficacy of PVCS LSP formulated in Montanide ISA-51 adjuvant in healthy malaria-naïve, and semi-immune volunteers are described.

\section{Methods}

\section{Ethics statement}

The study protocol was reviewed and approved by the Institutional Review Boards of the Malaria Vaccine and Drug Development Center (CECIV-MVDC) and Centro Médico Imbanaco (CMI \# 0992304-493-26202) (Appendix 1). The study complied with the Declaration of Helsinki principles, International Conference on Harmonization, Good Clinical Practices guidelines, and all pertinent Colombian regulations. All participants provided written informed consent (IC) and were advised that they were free to withdraw from the study at any time. Volunteers were excluded if they had diseases or medical conditions that would alter the vaccine's assessment or any condition that could increase the risk of adverse outcomes.

\section{Study design and participants}

This was a phase lla/b randomized, double-blind, comparative, controlled trial to evaluate the protective efficacy and safety of $P$. vivax CS protein formulated in Montanide ISA-51 adjuvant. Thirty-five healthy, Duffy positive $(\mathrm{Fy}+)$ men and non-pregnant women (19-44 years of age) were recruited from a larger group ( $n=121)$ and allocated into two groups: healthy malaria-naïve $(n=17)$ and malaria semi-immune $(\mathrm{n}=18)$ volunteers previously exposed to $P$. vivax. Participants were randomly (simple) assigned in a 2:1 ratio. A blinded data manager controlled the allocation to receive the vaccine (Experimental; Exp, $n=25$ ) or placebo (Control; Ctrl, $\mathrm{n}=10$ ) (Figure 1). Access to the randomization code was strictly controlled at the pharmacy. The naïve group was further divided into $\operatorname{Exp}(n=12)$ and $\operatorname{Ctrl}(n=5)$ and the semi-immune group into $\operatorname{Exp}(n=13)$ and $\mathrm{Ctrl}(\mathrm{n}=5)$. Naïve volunteers were from Cali (Colombia), a non-malaria endemic 
city, with eligibility based on no history of malaria and negative serology against $P$. vivax blood stages. Semi-immune volunteers were recruited from Buenaventura, a low to moderate malaria-endemic area of Colombia; eligible volunteers should have had a history of malaria and antibodies against $P$. vivax blood stages with titers higher than $1: 20$ by IFAT or higher than 1:200 by ELISA using a recombinant $P$. vivax MSP1 (Pv200L) protein [21].

\section{Vaccines}

The three LSP (N, R, and C) synthesized under good laboratory practices (GLP) conditions at the Biochemistry Institute, University of Lausanne, Switzerland, were packaged, lyophilized, and then tested for sterility and apyrogenicity. As previously described [13], the $\mathrm{N}$ polypeptide corresponded to N-terminal amino acids (aa) 20-96, the $\mathrm{C}$ peptide to C-terminal aa 301-372. In contrast, the R peptide VK210 (type I) corresponded to a construct based on the first central non-peptide repeat (aa 96-104) in tandem three times and collinearly linked to a universal T-cell epitope (ptt-30) derived from tetanus toxin [22-24]. A 1:1:1 peptide mixture (50 $\mu \mathrm{g} /$ each peptide) was emulsified in Montanide ISA-51 (Seppic Inc., Paris, France) in the same proportion according to manufacturer recommendations on the same day of subject immunizations. Saline solution (Baxter, Deerfield, IL) was emulsified with the same adjuvant and used as a placebo.

\section{Interventions}

The primary outcome measure was to assess the $P$. vivax CS LSP vaccine's protective efficacy to the $P$. vivax $\mathrm{CHMI}$ in malaria-naïve and semi-immune volunteers, and the secondary outcome, the immune response associated with protection. Eligible participants were enrolled to receive three doses of vaccines at months 0,2 , and 6 containing the mixture of LSP $(150 \mu \mathrm{g} / \mathrm{dose})$ or placebo, formulated in the Montanide ISA-51 adjuvant by i.m. injection in the deltoid muscle with a volume of $0.5 \mathrm{~mL}$. The first immunization dose contained a mixture of peptides $\mathrm{N}$ and $\mathrm{C}$ only $(50 \mu \mathrm{g} /$ peptide; total dose $100 \mu \mathrm{g} / \mathrm{dose})$,

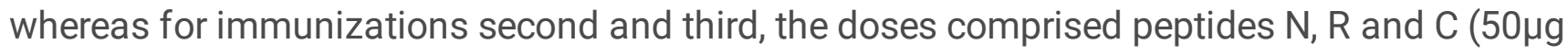
peptide/dose; a total of $150 \mu \mathrm{g}$ protein/dose). Control groups were immunized with the placebo (saline solution) formulated in the same adjuvant's total volume. Vaccines were prepared by staff researchers not involved with patient care.

\section{Safety}

During the hour following immunization, volunteers were under direct medical supervision to detect any adverse reaction to the vaccine injection, after which a physical examination was performed. Eight hours after vaccination, volunteers' physical status was assessed by a telephone call. Also, a personal follow-up was performed one week before the following immunizations. Clinical laboratory tests were performed to evaluate vaccine tolerability and safety at months $0,1,2,3,6,7,9$, and 10. Blood samples were collected to determine Fy blood group, G6PD deficiency test, complete blood count (CBC), prothrombin time (PT), partial thromboplastin time (PTT), alanine aminotransferase (ALT), aspartate aminotransferase (AST), bilirubin, alkaline phosphatase, blood urea nitrogen (BUN), creatinine, and a pregnancy test in women. 
Volunteers were also under observation for one hour after the $\mathrm{CHMl}$ and then by phone monitoring eight hours after and once a day until day four. Volunteers were then evaluated daily for clinical manifestations and microscopic patent parasitemia from days five to 30 after challenge and later on, every second day until day 60. Two experienced, independent microscopists evaluated parasitemia by counting the number of asexual $P$. vivax parasites per 400 white blood cells (WBC), assuming normal WBC counts (8000 cell $/ \mathrm{mL}$ ). Samples were considered negative after observing 200 microscopic fields, and qPCR was performed subsequently for retrospective analysis. Adverse Events (AE) were recorded, graded and classified according to FDA recommendations [25].

\section{Sporozoite production}

Whole blood (15mL) was collected by venipuncture (Vacutainer tubes, Becton Dickinson, NJ, USA) from patients diagnosed with VK210 P. vivax in Leticia, Colombia, and used to infect colonized Anopheles albimanus mosquitoes. Fed mosquito batches were dissected and microscopically examined for the presence of oocysts in the midgut (day 7) and sporozoites in salivary glands (day 14) as previously described [26]. Only batches with $\geq 60 \%$ sporozoite infection rates were considered acceptable for $\mathrm{CHMI}$ to evaluate the protective efficacy. CHMI was performed three months after the last immunization by volunteer's exposure to 2-5 P. vivax infected-mosquito bites, as previously described $[15,16]$. After humanbiting, each mosquito's dissection confirmed blood in midguts and sporozoites in salivary glands [27].

\section{Humoral response}

Antibody response was assessed using blood samples collected on months $0,1,2,3,6,7$ and 10 and measured by enzyme-linked immunosorbent assay (ELISA) using as antigen the $\mathrm{N}$, $\mathrm{R}$, or $\mathrm{C}$ peptides $(1 \mu \mathrm{g} / \mathrm{mL})$, as described previously [11]. Controls were selected from a pool of sera from semi-immune blood donors (positive ctrl) and a pool of sera from malaria-naïve donors (negative ctrl). Also, peptidespecific lgG isotypes were determined using sera collected at months 0,7 , and 10 from immunized volunteers by ELISA [13]; titers $<1: 200$ were considered negative. In addition, anti-LSP antibodies' parasite recognition was determined at 0-, 7-, and 10-months using an immunofluorescence assay (IFAT) as described before [11].

\section{IFN-g ELIspot production}

Peripheral blood mononuclear cells (PBMC) collected at months $0,1,2,3,6,7$, and 10 were separated from whole blood using Ficoll-Histopaque (Sigma-Aldrich, St. Louis, MO) density gradients and used to determine the IFN-y-producing cells as previously described [12]. Fresh PBMCs ( $4 \times 10^{5} /$ well) were mixed with $10 \mu \mathrm{g} / \mathrm{mL}$ of each LSP, and after $40 \mathrm{~h}$ culture, spots were counted with an ELISpot reader (AID Autoimmun Diagnostika GmbH, Germany), and the results expressed as the mean number of IFN- $y$ spotforming cells ( $\mathrm{sfc}$ ) per $10^{6} \mathrm{PBMC}$. Volunteers were considered responders if the number of sfc in their samples have increased from their baseline level (before immunization on day 0 ); any increase $\geq 5 \mathrm{sfc}$ was considered positive [12]. 


\section{Statistical analysis.}

Data were collected and managed using REDCap (Nashville, TN, USA) electronic data capture tools, analyzed using SPSS version 16.0 software (SPSS Inc., Chicago, IL, USA), and plotted using Graph Pad Prism version 6.0 (GraphPad Software, San Diego, California, USA). The main outcome evaluated the frequency of $P$. vivax infection in volunteers vaccinated with PVCS LSP formulated in Montanide ISA-51. The sample size was calculated with a confidence level of $95 \%$, an error of $5 \%$ and an estimated prevalence of the Fy+ genotype of $30 \%$ with a population census of 5,603 in afro-descendent subjects from Buenaventura and 78\% from Cali [28]. All naïve and semi-immune participants were Fy+. Immunization's protective efficacy was assessed at a $5 \%$ significance level and $80 \%$ power. Nominal variables were analyzed using descriptive statistics. Mann-Whitney $\mathrm{U}$ or the Kruskal-Wallis tests were used as needed. Fisher's exact test was used to compare proportions. Spearman's rank correlation was used to assess the correlation between numeric variables. Incubation and prepatent periods were determined by microscopy and qPCR and visualized using Kaplan-Meier estimator. Antibody titers and IFN-g production were compared among groups at several points in the study using Wilcoxon signed-rank and the Kruskal-Wallis test. Two-tailed, non-parametric $p$ values $\leq 0.05$ were considered significant.

\section{Results}

Volunteers enrollment and retention: Aiming to enroll 52 volunteers from malaria-endemic and nonendemic regions, a total of 121 subjects were asked to participate in the trial. After 86 were excluded, 35 volunteers were eligible and enrolled. Seventeen volunteers were allocated to the naïve group (12 Exp +5 Ctrl) and 18 to the semi-immune groups (13 Exp $+5 \mathrm{Ctrl})$. All 35 volunteers were immunized with the PVCS LSP vaccine or placebo formulated in Montanide ISA-51. After the first immunization, two volunteers withdrew (one from the naïve and one from the semi-immune groups). One more volunteer from the semi-immune group had to be dropped-out because of a diabetes mellitus diagnosis considered an SAE not-related to the immunization. Therefore, for the rest of the study (immunizations 2 and 3 , and $\mathrm{CHMI})$, the groups were composed of $(11 \mathrm{Exp}+5 \mathrm{Ctrl})$ in each naïve and semi-immune groups (Figure 1). The mean age at enrollment was 31 years old (19-44 range). The median range was 30 years old (19-44 range) for women and 32 years old (20-43 range) for men.

\section{Vaccine and CHMI safety}

The vaccine was safe and well-tolerated. Mild (grade 1) to moderate (grade 2) local pain was the most frequently $(75 \%)$ reported $A E$ during vaccination (24/32), followed by headache in $31.25 \%$ (10/32) and malaise in $31.25 \%$ (10/32), which resolved in all cases the next day. Pain occurred more frequently in the Exp (16 subjects) than in the Ctrl group (8 subjects). Fever, nausea, chills, diarrhea, and abdominal pain occurred at low frequencies (1-3 subjects) during the vaccination period (Table 1).

Mild to moderate biochemical or hematological laboratory-related AE were observed. Mild anemia (10.7$11.5 \mathrm{~g} / \mathrm{dL}$. Normal values: $>12 \mathrm{~g} / \mathrm{dL}$ ) occurred in two females (naïve group), and two (one male/one female) (semi-immune group) volunteers after the first immunization; however, all normalized before the 
CHMI. Transient low-level proteinuria (Grade 1-2) was observed after the second immunization in the naïve group but reached normal values in the following month. Two semi-immune group volunteers presented prolonged thromboplastin time (37.7 and $39.3 \mathrm{sec}$.; normal value: $25-35 \mathrm{sec}$.). After the third immunization, a volunteer showed glycosuria of $500 \mathrm{mg} / \mathrm{dL}$ and glycosylated hemoglobin of $8.1 \%$; (HbA1c: normal value: $5.7 \%$ ) and was diagnosed with diabetes mellitus not related to vaccination (Appendix 2). All laboratory tests for the remaining participants were within the normal range during the study period. The $\mathrm{CHMI}$ was well tolerated, and minor AEs were recorded. No SAEs related to malaria infection were recorded.

\section{Vaccine Immunogenicity}

Positive ELISA using the $\mathrm{N}$ - and C-peptides indicated seroconversion of all 22 naïve and semi-immune volunteers after the first immunization. However, lower reactivity was observed in the semi-immune than in the naïve group. The second immunization induced a moderate boosting of antibodies to the amino $(\mathrm{N}-)$ and carboxyl (C-) fragments, as well as priming of the response to the repeat ( $\mathrm{R}$ ) region; however, only the naïve group displayed a significant increase ( $p$ 0.093). After the third immunization, a slight but significant boosting of antibodies to the three fragments was observed in both the naïve and semiimmune groups; however, this antibody increase was better in the naïve than in the semi-immune group (N-peptide, $p<0.045 ; R-, p<0.01 ; C-, p<0.016)$. All control volunteers remained seronegative during the immunization phase (Figure 2).

Single-cell IFN-g production by PBMC was similar in all volunteers from the experimental groups (naïve $p=0.66$; semi-immune $p=0.99$ ) with increased IFN- $y$ production during the immunization phase. However, after the third immunization, a significant difference was found between naïve and semi-immune groups IFN-g production in response to stimulation with the $\mathrm{N}$ terminal peptide $(p=0.002)$ and the $\mathrm{C}$ terminal one $(p=0.0194)$. At this time, IFN- $\gamma$ production in response to the R-peptide stimulation (post-second immunization) did not show any significant difference between naïve and semi-immune groups $(p=0.5332)$. Finally, there was no boosting of the IFN-y response upon sporozoites CHMl; instead, there was a decrease of this cytokine's levels in both groups after $\mathrm{CHMI}$. This decrease was highly significant in the naïve group (N-peptide, $p=0.0001, R-, p=<0.0001$, and $C-, p=0.0367$ ) whereas it was non-significant in the semi-immune group (N-peptide, $p=0.767, R-p=0.189$, and $C-, p=0.293$ ) (Figure 4).

\section{Vaccine efficacy}

The study's primary outcome was the $P$. vivax infection's frequency as a measure of the PVCS LSP vaccination's protective efficacy against $P$. vivax infection in malaria-naïve and semi-immune volunteers. From days 14 to 19 after CHMI, symptoms consistent with malaria like fever, chills, headache, and profuse sweating were shown by $6 / 11$ and 5/5 volunteers of the naïve Exp and naïve Ctrl groups, respectively, and 9/11 and 4/5 volunteers of the semi-immune Exp and semi-immune Ctrl groups respectively, which lasted until malaria treatment. Protective efficacy determined by the presence of patent microscopic parasitemia was observed in five of 11 naïve Exp volunteers (42\%) and four of 11 semi-immune Exp volunteers (36\%), who did not develop parasitemia during the 60-day follow-up. One 
volunteer from the semi-immune Ctrl group did not develop parasitemia. In contrast, the remaining four controls were confirmed to develop blood-stage $P$. vivax parasite infections between days 15-17. All nonprotected volunteers from experimental groups developed patent infection between 12-19 days after CHMI with a mean prepatent period of 15.71 days (range 14-16 days) in the naïve Exp and 15.88 days (range 12-19 days) in the semi-immune Exp group (Figure 2). Survival analysis showed that sterile protection was observed in four of 11 naïve volunteers $(36.6 \%)$ and three of the 11 semi-immune volunteers $(27.3 \%)$ who did not show parasitemia after 60 days follow-up.

\section{Discussion}

This phase lla/b vaccine trial provides evidence that the immunization of malaria naïve and semiimmune volunteers with mixtures of LSP derived from PVCS formulated in Montanide ISA-51 is safe, welltolerated, immunogenic, and induces sterile protection. To our knowledge, this is the first report of a comparative $P$. vivax clinical trial inducing sterile protection in naïve (36.6\%), and semi-immune (27.3\%) volunteers against $\mathrm{CHMl}$, as determined by the absence of malaria symptoms or patent parasitemia during the 60 days of close follow-up.

The safety, tolerability and immunogenicity observed are consistent with previously reported vaccination studies with similar P. vivax CS derived LSP formulated individually in Montanide ISA-720 [11] or as peptide mixture in Montanide ISA-720 or Montanide ISA-51 [12]. Furthermore, our results also show safety and reproducibility of the sporozoite induced $\mathrm{CHMI}$ [16] as well as the efficacy of the antimalarial treatment to radically cure the $P$. vivax infection as determined by the absence of clinical relapses during the close follow-up period (60 days) in any of the study participants who required anti-parasite treatment upon patent malaria confirmation. However, one of the semi-immune Ctrl group participants developed parasitemia (SAE) about two months after returning to the endemic areas, probably due to reinfection. It is striking that one of the semi-immune Ctrl group volunteers did not develop parasitemia. It might have been due to mosquito failure to infect the volunteer, as observed in a previous $\mathrm{CHMI}$ trial [15], or that the volunteer could have taken antimalarials drugs or beverages, which could not be documented.

A previous trial using the VMP001 PVCS vaccine formulation based on a recombinant $P V C S$ protein expressed in E. coliformulated in AS01-B (GSK) showed it safe, well-tolerated, and immunogenic with a slight delay in the prepatent period [19]. In that dose-escalation (15, 30 and $60 \mu \mathrm{g} / \mathrm{dose})$ trial, volunteers with higher immunogen doses presented a delay (two days) in parasite patency in $59 \%$ of the vaccinees. Here control volunteers, both naïve and semi-immune, developed a similar mean prepatent period (naïve $\operatorname{Exp}=15.8$ day) (semi-immune $\operatorname{Exp}=16.8$ days), and none of the volunteers in the experimental groups (naïve Exp and semi-immune Exp) developed significant partial delays. Another remarkable observation was the semi-immune volunteers' apparent hypo-responsiveness to the vaccine, as indicated by the significant differences observed between the naïve and semi-immune Exp groups in the specific IgG and IFN-g responses. Recent malaria vaccine trials have also demonstrated differential immune responsiveness to pre-erythrocytic vaccine candidates ( $P f$-RTSS, R21) in inhabitants from malariaendemic and non-endemic areas [29], suggesting that individuals from malaria-endemic regions, either 
actively infected or not, display an altered basal immune status with a paucity of regulatory mechanisms and altered memory cell function leading to lower responsiveness to vaccines [30-32]. In addition, it is likely that other hosts- and environmental-factors such as sex, ethnicity, nutrition, malaria endemicity, and others influence host-immune response and immunity to malaria. Another intriguing observation was the significant decreasing trend of IFN- $\gamma$ secretion displayed by most individuals exposed to sporozoite $\mathrm{CHMI}$ (Figure 3). This striking result might be related to the volunteers' exposure to mosquito's saliva as some studies have shown that after biting, saliva can trigger different effects on humans' immune cells in mice grafted with human hematopoietic stem cells directly by the Th1/Th2 response [31, 33].

Importantly, AEs related to vaccination occurred with similar frequency in all groups and most volunteers; these were limited to local, transient pain at the vaccine injection site, in agreement with those reported for commercially available vaccines [38], with systemic AEs consisting of malaise and headache [34]. This mild reactogenicity has already been reported in previous studies using the same immunogen in $>100$ naïve volunteers [22,35]. In contrast, unacceptable reactogenicity previously described in HIVderived (peptide cocktails) and Pfs-25 (recombinant protein) vaccines formulated in Montanide adjuvant was not observed here [36]. Only minor reactogenicity, consistent with previous phase I vaccine trials conducted with the same formulations, was observed [12]. These results agree with a significant number of vaccine trials conducted on different antigens formulated in Montanide ISA-51 with or without or with minor reactogenicity $[37,38]$. The inclusion of semi-immune volunteers in this study represented an exceptional logistic challenge. Despite volunteers being from a malaria-endemic region located only 70 miles from the research center in Cali, the numerous visits during enrolment and vaccination periods and their retention post-CHMI provided essential hurdles to completing the study. Although semi-immune volunteers were requested to be outside the endemic area for the parasitemia follow-up, some decided to return to their homes about a month after $\mathrm{CHMl}$, making follow-up more demanding. Therefore, one of the limitations of the study was volunteer retention. Nevertheless, it provided a unique opportunity to directly assess the vaccine candidate's protective efficacy in volunteers previously exposed to natural P.vivax infections, representing a proof-of-principle for further Phase llb trials.

This study sample is not representative of the whole population of individuals living in the country's malaria-endemic areas; thus, a new trial with a larger volunteer group is being conducted in parallel in malaria-endemic and non-endemic regions (NCT 04739917). This upcoming study will also address ethnicity, the potential immunological imbalance caused by permanent exposure to malaria parasites, mosquito bites and other environmental factors in endemic areas to identify immune-signatures that may explain and predict the hypo-responsiveness to malaria vaccination and its effect on vaccine-elicited immunity.

\section{Declarations}

\section{Data availability}


Data that support the study is into the REDCap database, available from the corresponding author upon reasonable request. This trial is registered on ClinicalTrials.gov under the identifier NCT02083068

\section{Acknowledgments}

We thank the volunteers from Cali and Buenaventura for their invaluable collaboration in the study. As well as the parasite's donors from Leticia (Colombia), the continuous support from Dr. Armando Gonzalez-Materon, C.E.O. of the CMI, and the technical support from N. Cespedes and A. Vallejo, John García, Maria Isabel Arce and Laureano Mestra. To Seppic Inc., Paris, France for kindly providing the adjuvant.

\section{Funding}

This trial was funded by Colciencias (grant 529-2009), NHLBI (grant RHL086488 A), and the MVDC/CIV Foundation.

\section{Competing Interest}

The authors declare no competing interest.

\section{Author contributions}

Conceptualization: M.A.H, S.H.

Formal analysis: M.A.H., G.C., S.M.H, A.C., XG.

Investigation: M.A.H., S.H., G.C., S.M.H., M.L.D., M.A.C., J.H.G., A.C., X.G.

Methodology: MAH, SH, GC, SMH, XG, AC, JHG, JO, JCD, AP.

Project administration: M.A.H., S.H.

Resources: G.C., M.A.H., S.H.

Supervision: M.A.H., S.H., J.O.

Validation: M.A.H., SH., J.O.

Visualization: M.A.H, S.H., GC, S.MH.

Writing: M.A.H, S.H., GC, S.M.H.

\section{Co-authors Emails:}

Myriam Arévalo-Herrera: marevalo@inmuno.org

Michelle Larmat-Delgado: michellelarmat@hotmail.com 
1. Alejandra Caicedo: mcaicedog@unal.edu.co

Juliana Henao-Giraldo: juliana.henao.giraldo@gmail.com

Angélica Castellanos: angelica.castellanos@correounivalle.edu.co

Xiomara Gaitán:alejandra.gaitan@inmuno.org

Sonia M. Herrera: sonia.herrera@inmuno.org

José Oñate : millanonate@gmail.com

Jean-Christophe Devaud:Jean-Christophe.Devaud@chuv.ch

André Panatier : André.Panatier@chuv.ch

Giampietro Corradin: giampietro.corradin@unil.ch

\section{References}

1. WHO, Malaria progress report. 2020, WHO: Geneva.

2. Gething, P.W., et al., A long neglected world malaria map: Plasmodium vivax endemicity in 2010. PLoS Negl Trop Dis, 2012. 6(9): p. e1814.

3. Matuschewski, K., Vaccines against malaria-still a long way to go. FEBS J, 2017. 284(16): p. 25602568.

4. Asante, K.P., et al., Safety and efficacy of the RTS,S/ASO1E candidate malaria vaccine given with expanded-programme-on-immunisation vaccines: 19 month follow-up of a randomised, open-label, phase 2 trial. Lancet Infect Dis, 2011. 11(10): p. 741-9.

5. Vandoolaeghe, P. and L. Schuerman, [The RTS,S/ASO1 malaria vaccine in children aged 5-17 months at first vaccination]. Pan Afr Med J, 2018. 30: p. 142.

6. Laurens, M.B., RTS,S/ASO1 vaccine (Mosquirix): an overview. Hum Vaccin Immunother, 2020. 16(3): p. $480-489$.

7. Cotton, M., The Mosquirix (RTS.S) malaria vaccine. Trop Doct, 2020. 50(2): p. 107.

8. Valencia, S.H., et al., Platform for Plasmodium vivax vaccine discovery and development. Mem Inst Oswaldo Cruz, 2011. 106 Suppl 1: p. 179-92.

9. Arevalo-Herrera, M., et al., Preclinical vaccine study of Plasmodium vivax circumsporozoite protein derived-synthetic polypeptides formulated in montanide ISA 720 and montanide ISA 51 adjuvants. Am J Trop Med Hyg, 2011. 84(2 Suppl): p. 21-7.

10. Herrera, S., et al., Antigenicity and immunogenicity of multiple antigen peptides (MAP) containing P. vivax CS epitopes in Aotus monkeys. Parasite Immunol, 1997. 19(4): p. 161-70. 
11. Herrera, S., et al., Safety and elicitation of humoral and cellular responses in colombian malaria-naive volunteers by a Plasmodium vivax circumsporozoite protein-derived synthetic vaccine. Am J Trop Med Hyg, 2005. 73(5 Suppl): p. 3-9.

12. Herrera, S., et al., Phase I safety and immunogenicity trial of Plasmodium vivax CS derived long synthetic peptides adjuvanted with montanide ISA 720 or montanide ISA 51. Am J Trop Med Hyg, 2011. 84(2 Suppl): p. 12-20.

13. Cespedes, N., et al., Antigenicity and immunogenicity of a novel Plasmodium vivax circumsporozoite derived synthetic vaccine construct. Vaccine, 2014. 32(26): p. 3179-86.

14. Arevalo-Herrera, M., et al., Protective Efficacy of Plasmodium vivax Radiation-Attenuated Sporozoites in Colombian Volunteers: A Randomized Controlled Trial. PLoS Negl Trop Dis, 2016. 10(10): p. e0005070.

15. Herrera, S., et al., Successful sporozoite challenge model in human volunteers with Plasmodium vivax strain derived from human donors. Am J Trop Med Hyg, 2009. 81(5): p. 740-6.

16. Herrera, S., et al., Consistent safety and infectivity in sporozoite challenge model of Plasmodium vivax in malaria-naive human volunteers. Am J Trop Med Hyg, 2011. 84(2 Suppl): p. 4-11.

17. Gordon, D.M., et al., Safety and immunogenicity of a Plasmodium vivax sporozoite vaccine. Am J Trop Med Hyg, 1990. 42(6): p. 527-31.

18. Herrington, D.A., et al., Safety and immunogenicity of a recombinant sporozoite malaria vaccine against Plasmodium vivax. Am J Trop Med Hyg, 1991. 45(6): p. 695-701.

19. Yadava, A., et al., A novel chimeric Plasmodium vivax circumsporozoite protein induces biologically functional antibodies that recognize both VK210 and VK247 sporozoites. Infect Immun, 2007. 75(3): p. $1177-85$.

20. Bennett, J.W., et al., Phase 1/2a Trial of Plasmodium vivax Malaria Vaccine Candidate VMP001/AS01B in Malaria-Naive Adults: Safety, Immunogenicity, and Efficacy. PLoS Negl Trop Dis, 2016. 10(2): p. e0004423.

21. Valderrama-Aguirre, A., et al., Polymorphism of the Pv200L fragment of merozoite surface protein-1 of Plasmodium vivax in clinical isolates from the Pacific coast of Colombia. Am J Trop Med Hyg, 2011. 84(2 Suppl): p. 64-70.

22. Corradin, G., et al., Malaria vaccine development using synthetic peptides as a technical platform. Adv Immunol, 2012. 114: p. 107-49.

23. Herrera, S., et al., Use of long synthetic peptides to study the antigenicity and immunogenicity of the Plasmodium vivax circumsporozoite protein. Int J Parasitol, 2004. 34(13-14): p. 1535-46.

24. Herrera, S., et al., Human recognition of T cell epitopes on the Plasmodium vivax circumsporozoite protein. J Immunol, 1992. 148(12): p. 3986-90.

25. FDA, U.S. Food and Drug Administration. Guidance for Industry. Toxicity Grading Scale for Healthy Adultand Adolescent Volunteers Enrolled in Preventive Vaccine Clinical Trials. Washington D.C., United States of America: U.S. Food and Drug Administration; 2007., U.S.F.a.D. Administration., Editor. 2007: U.S. 
26. Hurtado, S., et al., Regular production of infective sporozoites of Plasmodium falciparum and P. vivax in laboratory-bred Anopheles albimanus. Ann Trop Med Parasitol, 1997. 91(1): p. 49-60.

27. Solarte, Y., et al., Plasmodium vivax sporozoite production in Anopheles albimanus mosquitoes for vaccine clinical trials. Am J Trop Med Hyg, 2011. 84(2 Suppl): p. 28-34.

28. Vallejo, A.F., et al., High prevalence of sub-microscopic infections in Colombia. Malar J, 2015. 14: p. 201.

29. Kazmin, D., et al., Systems analysis of protective immune responses to RTS,S malaria vaccination in humans. Proc Natl Acad Sci U S A, 2017. 114(9): p. 2425-2430.

30. Mo, A.X.Y., et al., Understanding vaccine-elicited protective immunity against pre-erythrocytic stage malaria in endemic regions. Vaccine, 2020. 38(48): p. 7569-7577.

31. Vogt, M.B., et al., Mosquito saliva alone has profound effects on the human immune system. PLoS Negl Trop Dis, 2018. 12(5): p. e0006439.

32. Yap, X.Z., M.B.B. McCall, and R.W. Sauerwein, Fast and fierce versus slow and smooth: Heterogeneity in immune responses to Plasmodium in the controlled human malaria infection model. Immunol Rev, 2020. 293(1): p. 253-269.

33. Hopp, C.S. and P. Sinnis, The innate and adaptive response to mosquito saliva and Plasmodium sporozoites in the skin. Ann N Y Acad Sci, 2015. 1342: p. 37-43.

34. Boffito, M., et al., Safety, immunogenicity and efficacy assessment of HIV immunotherapy in a multicentre, double-blind, randomised, Placebo-controlled Phase Ib human trial. Vaccine, 2013. 31(48): p. 5680-6.

35. Arevalo-Herrera, M., C. Chitnis, and S. Herrera, Current status of Plasmodium vivax vaccine. Hum Vaccin, 2010. 6(1): p. 124-32.

36. Wu, Y., et al., Phase 1 trial of malaria transmission blocking vaccine candidates Pfs 25 and Pvs25 formulated with montanide ISA 51. PLoS One, 2008. 3(7): p. e2636.

37. Chiang, C.L., L.E. Kandalaft, and G. Coukos, Adjuvants for enhancing the immunogenicity of whole tumor cell vaccines. Int Rev Immunol, 2011. 30(2-3): p. 150-82.

38. van Doorn, E., et al., Safety and tolerability evaluation of the use of Montanide ISA51 as vaccine adjuvant: A systematic review. Hum Vaccin Immunother, 2016. 12(1): p. 159-69.

\section{Table 1}

Table 1. Number of volunteers reporting vaccine-related adverse events in experimental and control groups 


\begin{tabular}{|c|c|c|c|c|c|c|}
\hline & & Naïve & Placebo & Semi-immune & Placebo & \multirow[t]{2}{*}{ Total } \\
\hline \multirow[t]{2}{*}{ Adverse events } & \# doses & $\begin{array}{lll}\text { I } & \text { II } & \text { III }\end{array}$ & $\begin{array}{lll}\text { I } & \text { II } & \text { III }\end{array}$ & $\begin{array}{lll}\text { I } & \text { II } & \text { III } \\
\end{array}$ & $\begin{array}{|lll|}\text { I } & \text { II } & \text { III } \\
\end{array}$ & \\
\hline & $\mathrm{n}$ & $(11)$ & (5) & $(11)$ & (5) & 64 \\
\hline Local & & & & & & \\
\hline Injection site pain & & 21 & 21 & 23 & 22 & 24 \\
\hline Swelling & & 11 & & & & 2 \\
\hline Systematic & & & & & & \\
\hline Headache & & 2 & $\begin{array}{lll}1 & 1 & 1\end{array}$ & 11 & 1 & 10 \\
\hline Malaise & & 11 & 1 & 1 & 22 & 10 \\
\hline Fever & & 1 & 1 & 1 & 1 & 4 \\
\hline Nausea/Emesis & & 1 & & 1 & 1 & 5 \\
\hline Chills & & & & 2 & & 2 \\
\hline Diarrhea & & 2 & 1 & 1 & & 5 \\
\hline Abdominal pain & & 1 & & & 1 & 2 \\
\hline
\end{tabular}

\section{Figures}

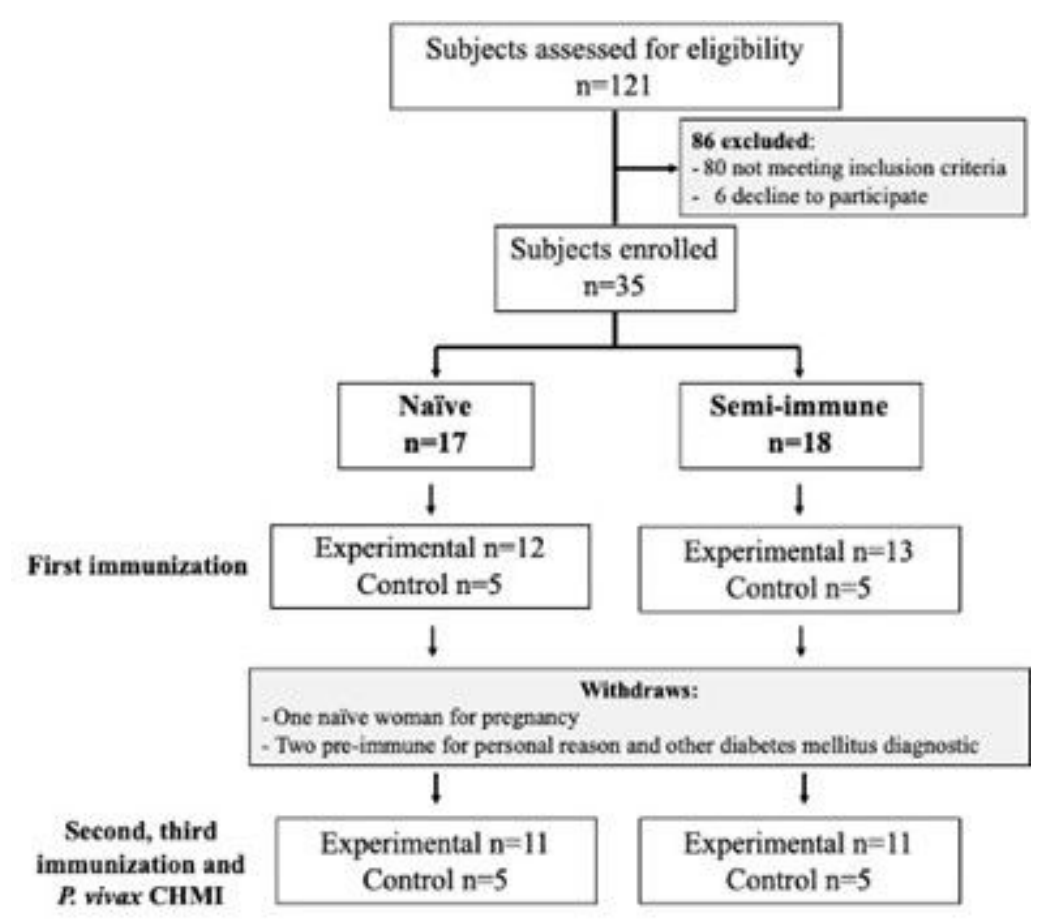

\section{Figure 1}

The number of individuals in the screening, immunization, and CHMI steps. 
Naïve group

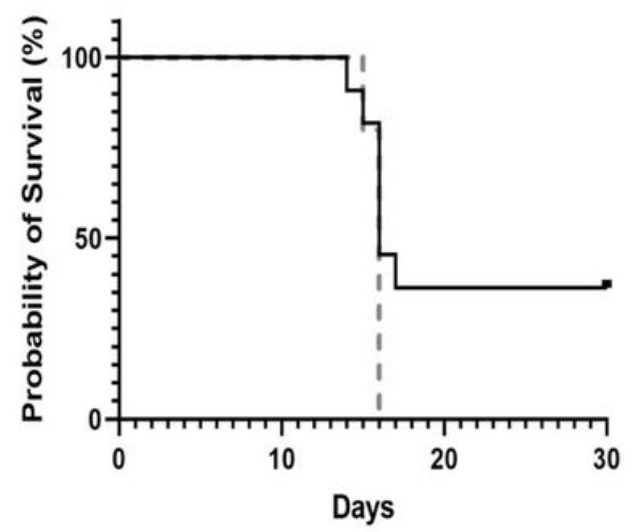

Semi-immune group

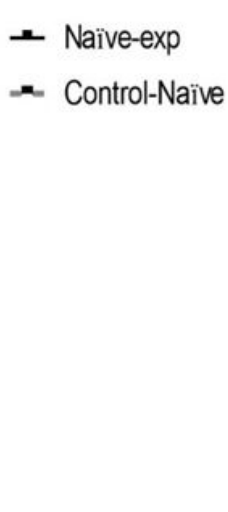

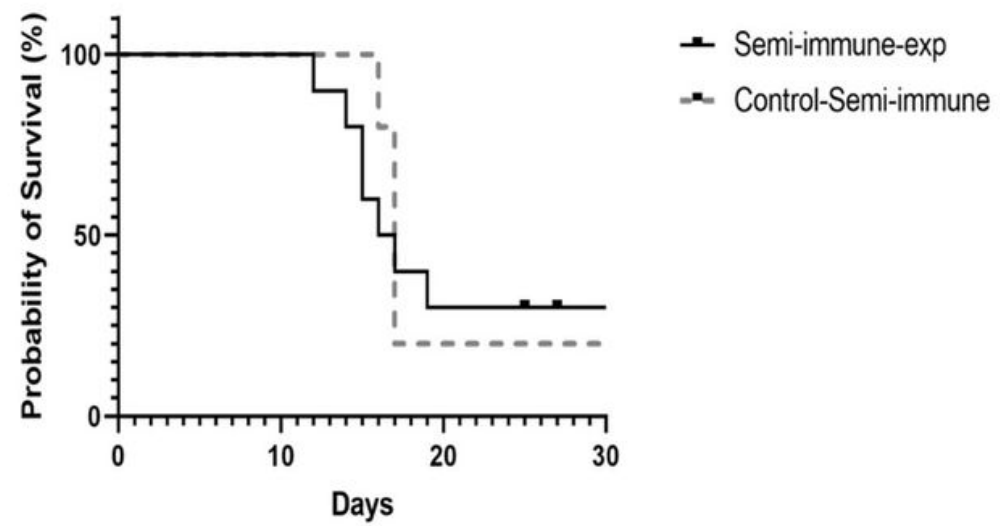

Figure 2

Protective efficacy Kaplan-Meier curves for Naïve and Semi-immune group. One volunteer of the semiimmune Ctrl group did not develop parasitemia.

Naĩve
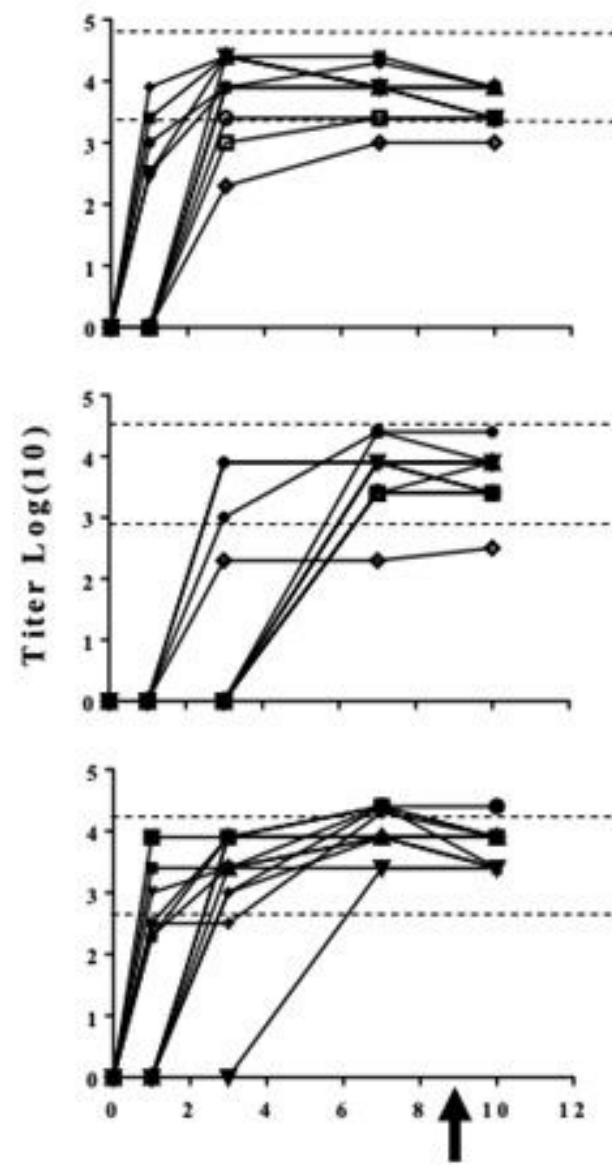

Semi-immune

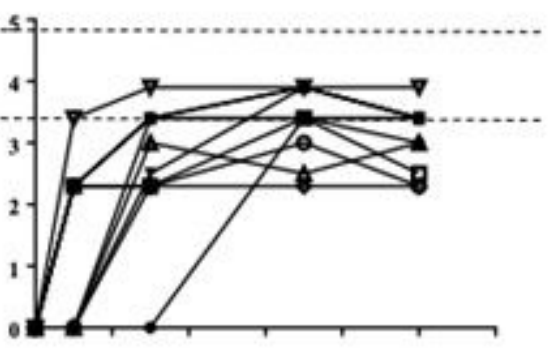

$\mathbf{N}$

$\mathbf{R}$

Months
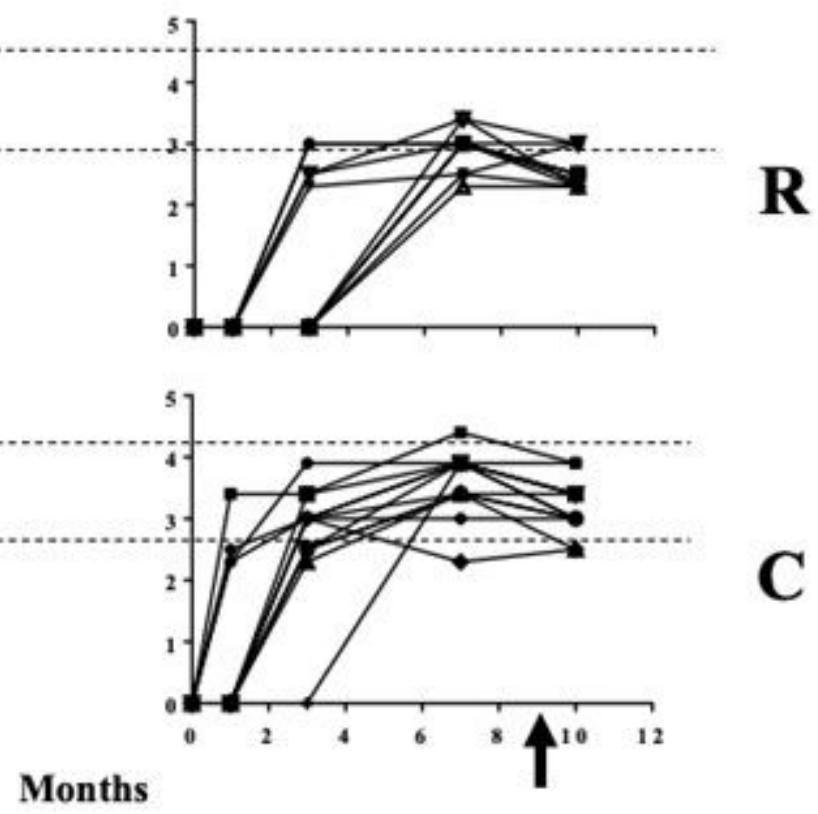

Figure 3 
IgG antibody response of volunteers vaccinated with a mixture of PvCS N, R and C peptides, $150 \otimes \mathrm{g} / \mathrm{mL} /$ dose formulated in Montanide ISA-51. Values are expressed as reactivity index (RI) defined as sample OD at 1:200 serum dilutions divided by the cut-off value. All participants seroconverted after the first vaccination, and none had a boosting effect observed after the second and third vaccination or $\mathrm{CHMI}$ (arrows)
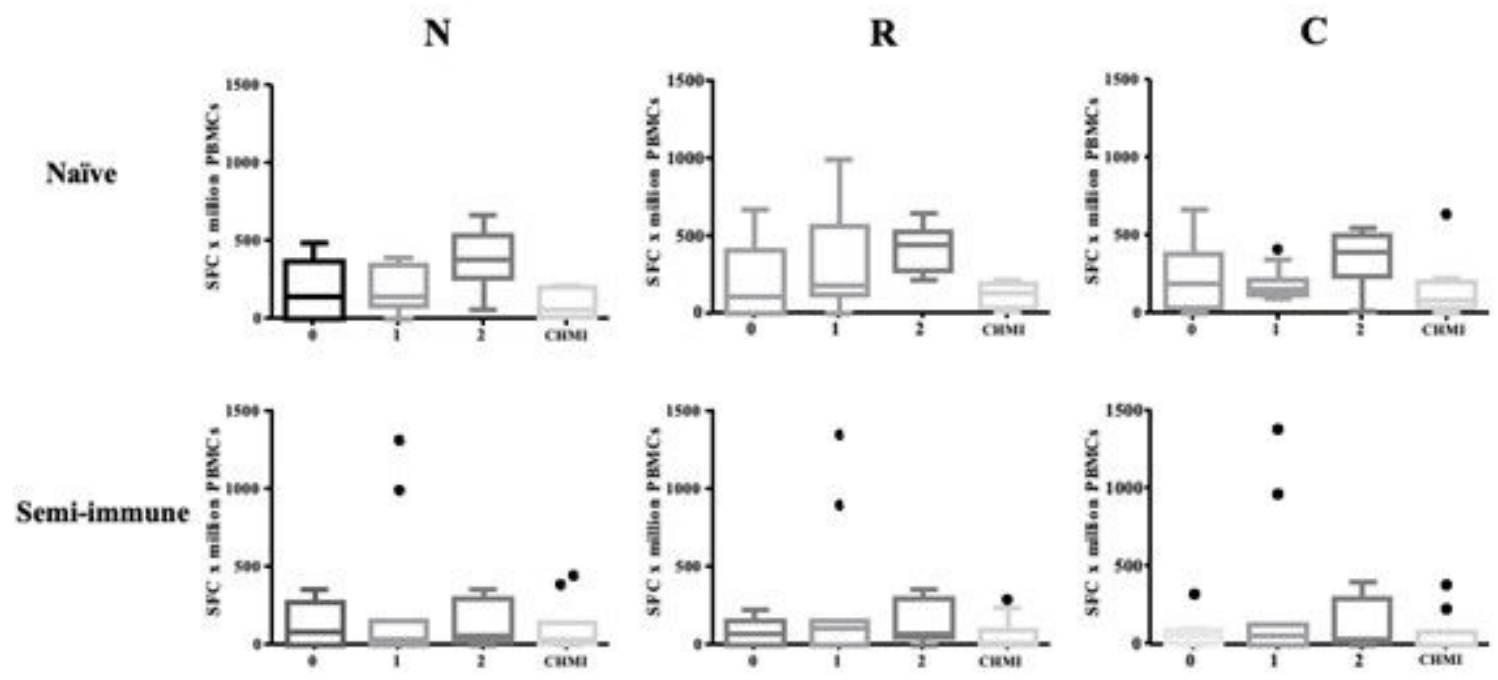

Immunization time and challenge

\section{Figure 4}

IFN- $\gamma$ production by PBMC stimulated with N, R and C peptides. The number of IFN- - -sfc/106 cells was evaluated by ELIspot using fresh PBMC from volunteers after each immunization cultures for $36 \mathrm{~h}$ in the absence or presence of individuals peptides. The results are expressed as the number of IFN- $\gamma-\mathrm{sfc} / 106$ cells PBMC of immunized volunteers.

\section{Supplementary Files}

This is a list of supplementary files associated with this preprint. Click to download.

- Appendix2ParaclinicaldataPvCSPhaselltrial29032110amshv.pdf

- Appendix1ProtocolfaselIPvCS.pdf 